\title{
The Use of Continuous Peritoneal Dialysis in Europe for the Treatment of Children with End-Stage Renal Failure: Data from the EDTA Registry
}

\author{
G. Rizzoni \\ Istituto di Ricerca Scientifica, Roma, \\ Italy
}

M. Broyer

J. H. H. Ehrich

N. H. Selwood

F. P. Brunner*

Hôpital Necker Enfants-Malades, Paris, France

Medizinische Hochschule Hannover, Federal Republic of Germany
UK Transplant Service, Bristol, United Kingdom

Department für Innere Medizin, Universität Basel, Switzerland

\author{
H. Brynger Department of Surgery 1, \\ Sahlgrenska Sjukhuset, Göteborg, \\ Sweden \\ S. R. Dykes St Thomas' Hospital, London, \\ United Kingdom \\ W. Fassbinder Städtische Kliniken Fulda, Federal \\ Republic of Germany \\ W. Geerlings Stichting Thuisdialyse Noord \\ Nederland, Haren, Netherlands \\ G. Tufveson Transplantation Unit, University \\ Hospital, Uppsala, Sweden \\ A. J. Wing St Thomas' Hospital, London, \\ United Kingdom
}

${ }^{*}$ Chairman

\begin{abstract}
The demographic data on the use of continuous peritoneal dialysis in Europe for children starting renal replacement therapy under the age of 15 years was obtained from data collected by the Registry of the European Dialysis and Transplant Association-European Renal Association (EDTA Registry) on individual patient questionnaires 1980-1986. Continuous ambulatory peritoneal dialysis (CAPD) and its variants appeared to be increasingly utilised as treatment for children with end-stage renal failure (ESRF) and
\end{abstract}

Correspondence and offprint requests to: Professor F. P. Brunner EDTA Registry, St Thomas' Hospital, London SE1 7EH, UK. accounted for approximately $25 \%$ of all renal replacement therapy (RRT). Important differences in its use in various European countries are demonstrated. The proportional contribution of CAPD to treatment was higher during the first year of RRT and gradually decreased thereafter. No significant sex differences existed in the use of this treatment. Approximately $70 \%$ of all patients on CAPD were older than 6 years of age, but it is in those under 6 years that the highest proportion are put on CAPD as first method of treatment for end-stage renal failure. The most common cause of abandonment of this treatment was peritonitis, which contributed $50 \%$ of the drop-out rate.

Key words: CAPD in children; EDTA Registry; Chronic dialysis treatment; Peritoneal dialysis; Demography 


\section{Introduction}

During the past decade there has been an increasing interest in the use of continuous ambulatory peritoneal dialysis (CAPD) for treatment of children with endstage renal failure (ESRF). The use of this mode of treatment is frequent in Canada and in the United States $[1,2]$. In Europe CAPD is used commonly in some and rarely in other countries [3]. This paper will evaluate the demographic data on the use of CAPD as a chronic dialysis therapy in Europe, from 1980 to 1986.

\section{Methods}

The methods of data collection employed by the EDTA Registry have been previously described [4]. This paper is based entirely on data derived from the patient questionnaires 1980-1986. The proportional contribution to therapy of CAPD and of the other modes of treatment at different intervals from start of RRT had been calculated using the so-called Selwood analysis [6].

\section{Results and Comments}

\section{Contribution of CAPD to the Treatment of Children with End-Stage Renal Failure in Europe and in Individual Countries}

The contribution of CAPD to the treatment of children, that is patients reaching ESRF before age 15, in Europe has been evaluated in three different ways. First, the use of CAPD as a first method of treatment has been studied amongst children commencing RRT between 1980 and 1986. Second, the total contribution of CAPD, measured in patient-months, to the maintenance of children by dialysis over the same 7-year period has been determined and, finally, the relative contribution of CAPD to the treatment of children over time has been assessed.

Figure 1 shows the proportion of children who commenced dialysis in each of the years 1980-1986 and whose first method of treatment was CAPD. This figure increased from $8.5 \%$ of 436 children accepted for treatment in 1980 to $23.6 \%$ of 504 children who commenced in 1986. In the intervening years there was a gradual increase in both the absolute numbers and the proportions of patients whose first form of treatment was CAPD.

Not only has CAPD become a more common choice of first treatment for children, but its total contribution to the maintenance of paediatric patients on dialysis has increased. Figure 2 shows the proportion of the total patient months of dialysis provided to children in each

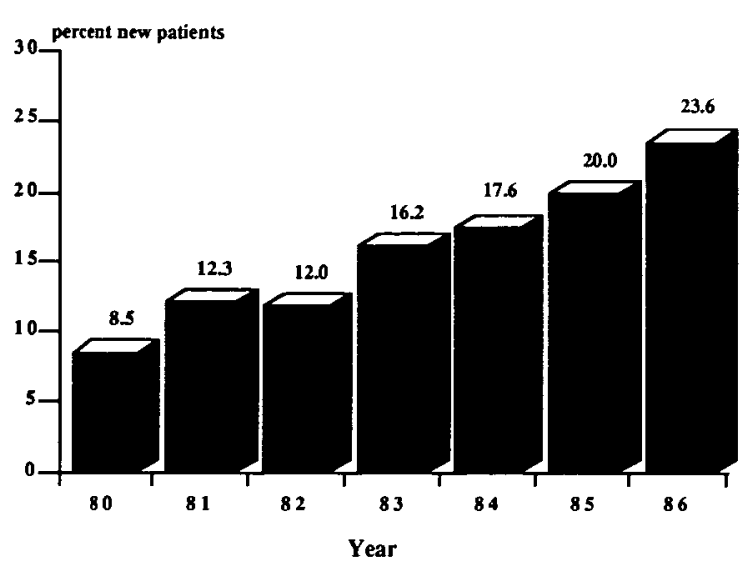

Fig. 1. Proportion (\%) of new patients commencing dialysis treatment in each of the years 1980-1986 who started CAPD as first method of treatment.

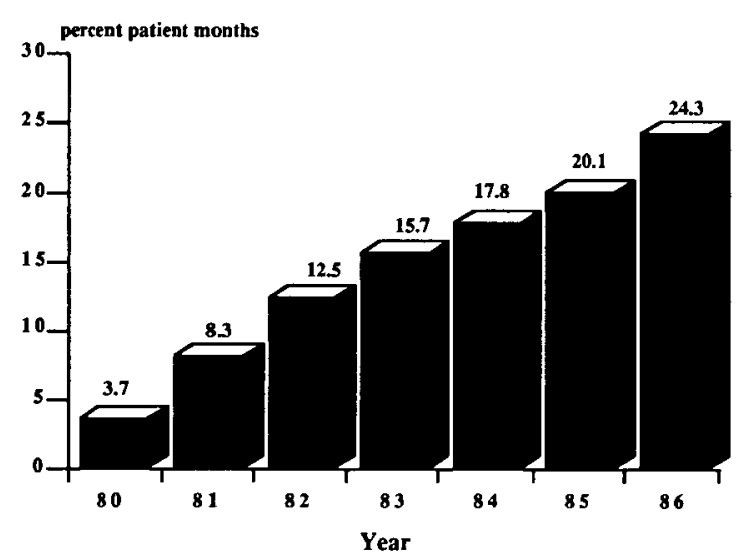

Fig. 2. Proportional contribution of CAPD in per cent patient-months to the maintenance of children on dialysis in Europe in each of the years $1980-1986$.

of the years 1980-1986 which was accounted for by CAPD. This analysis includes not only patients who commenced CAPD as first treatment in those years, but also those who were subsequently changed to this therapy. The results confirm the growing use of CAPD in children and show that by $198624.3 \%$ of patientmonths of therapy amongst these patients was provided by CAPD.

The total patient-months contribution of CAPD to treatment of children by dialysis has been analysed in a number of countries reporting to the Registry. Only those countries with at least 300 patient-months of total RRT recorded in paediatric patients during 1986 are included. The results are shown in Table 1 for each of the years 1981-1986. It is evident that there is great variation in policy for treating children with end-stage renal failure. In countries such as Egypt, the German Democratic Republic, and Yugoslavia, almost no CAPD in children was reported to the Registry between 
Table 1. Proportional contribution (\%) of CAPD to the maintenance of children on dialysis in selected European countries for each of the years 1981-1986

\begin{tabular}{lrrrrrr}
\hline Country & 1981 & 1982 & 1983 & 1984 & 1985 & 1986 \\
\hline & 4 & 8 & 9 & 8 & 0 & 13 \\
Austria & 7 & 8 & 5 & 9 & 7 & 18 \\
Belgium & 27 & 30 & 35 & 48 & 60 & 33 \\
Denmark & 0 & 0 & 0 & 0 & 0 & 10 \\
Egypt & 4 & 6 & 8 & 13 & 23 & 28 \\
Fed. Rep. Germany & 8 & 8 & 9 & 10 & 11 & 25 \\
France & 0 & 0 & 0 & 0 & 0 & 0 \\
German Dem. Rep. & 0 & 24 & 35 & 30 & 54 & 70 \\
Israel & 11 & 12 & 16 & 15 & 23 & 11 \\
Italy & 5 & 16 & 26 & 40 & 55 & 25 \\
Netherlands & 13 & 0 & 22 & 16 & 12 & 4 \\
Poland & 0 & 5 & 3 & 5 & 0 & 17 \\
Portugal & 9 & 5 & 7 & 8 & 13 & 29 \\
Spain & 2 & 6 & 59 & 53 & 21 & 50 \\
Sweden & 18 & 36 & 53 & 43 & 60 \\
Switzerland & 24 & 36 & 44 & 53 & 34 & 31 \\
United Kingdom & 26 & 39 & 45 & 50 & 34 & 7 \\
Yugoslavia & 0 & 0 & 0 & 0 & 7 & 0 \\
\hline
\end{tabular}

1981 and 1986, whereas in Denmark, Israel, Sweden, Switzerland, and the United Kingdom it contributed between $30 \%$ and $70 \%$ of all dialysis therapy in paediatric patients in 1986. In the Federal Republic of Germany, France, Italy, and Spain, CAPD made a smaller contribution to treatment in children, but the trend in the years 1981-1986 was of increasing use. This trend in individual countries is paralleled by the use of CAPD as first method of treatment; the proportion of new patients started directly on CAPD in each of the years 1980-1986 is shown for five European countries in Fig. 3.
The important variation in percentage from one year to another in the same country is partly due to the relatively small number of patients starting RRT and CAPD in a 12-month period. The great variation in policy between individual countries probably reflects a variety of factors. These include economic constraints, transplant activity, availability of already well-organised and functioning haemodialysis programmes, and persisting resistance among some doctors to the use of CAPD. A marked difference in national policies for the use of CAPD was also previously reported in adult patients by the Registry [5].

A special methodology has been developed for the Registry to analyse the relative contribution of different forms of RRT after first treatment. This so-called Selwood analysis describes the proportions of patients on different forms of treatment at specified intervals after first RRT [6,7]. The results give an indication of the probability that the patient will be on a particular form of treatment at a specified interval after first therapy. It takes account of patients who come onto a particular treatment or leave it, as well as those who die. The results of such analyses are shown for paediatric patients who commenced therapy in the years 19801986 in Fig. 4. The proportion of live patients on different forms of treatment at specified intervals after first RRT are given as percentages. At the start of therapy, hospital haemodialysis accounted for $68 \%$ of treatment, intermittent peritoneal dialysis (IPD) $11 \%$, CAPD $17 \%$, and $5 \%$ were grafted without prior dialysis. During the third year after the start of therapy the pattern had altered considerably, with transplant and hospital haemodialysis each contributing approximately

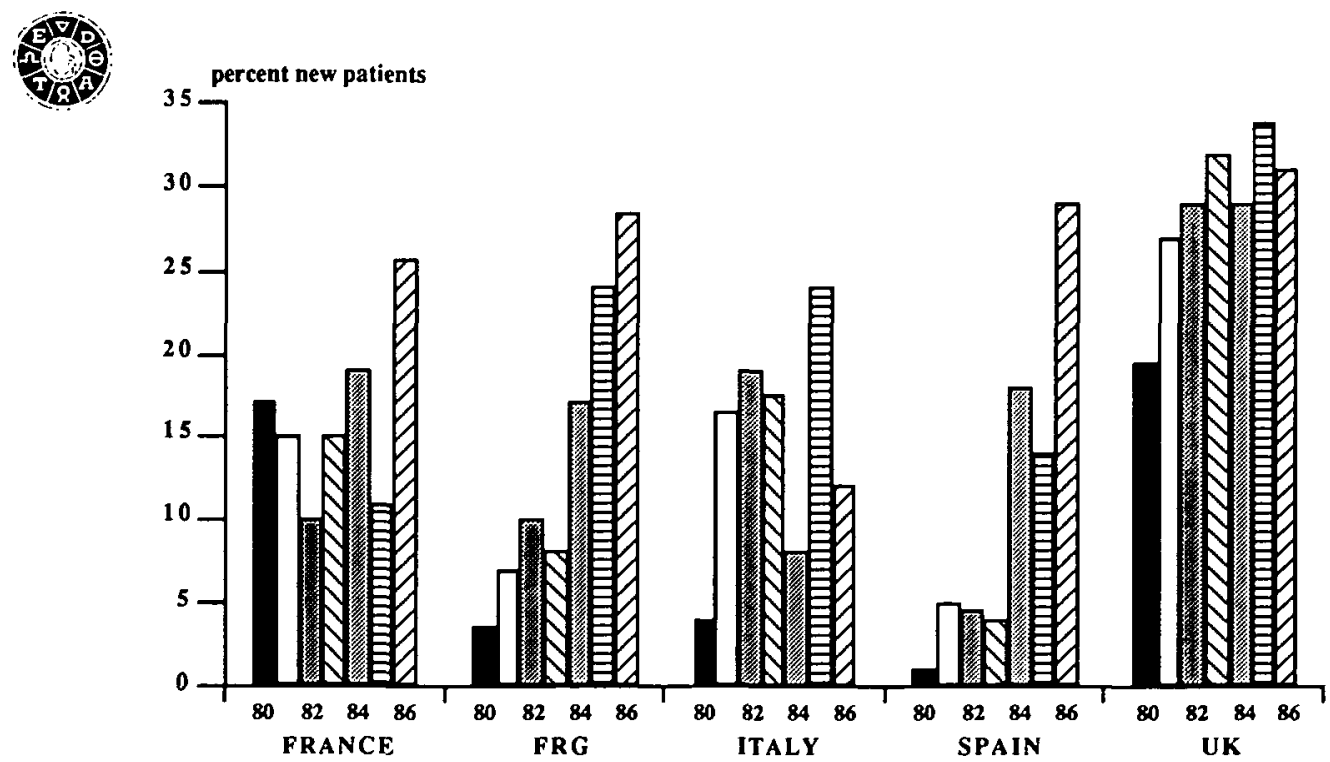

Fig. 3. Proportion of children commencing RRT in each of the years 1980-1986 who started CAPD as first method of treatment in France, the Federal Republic of Germany (FRG), Italy, Spain, and the United Kingdom (UK). 


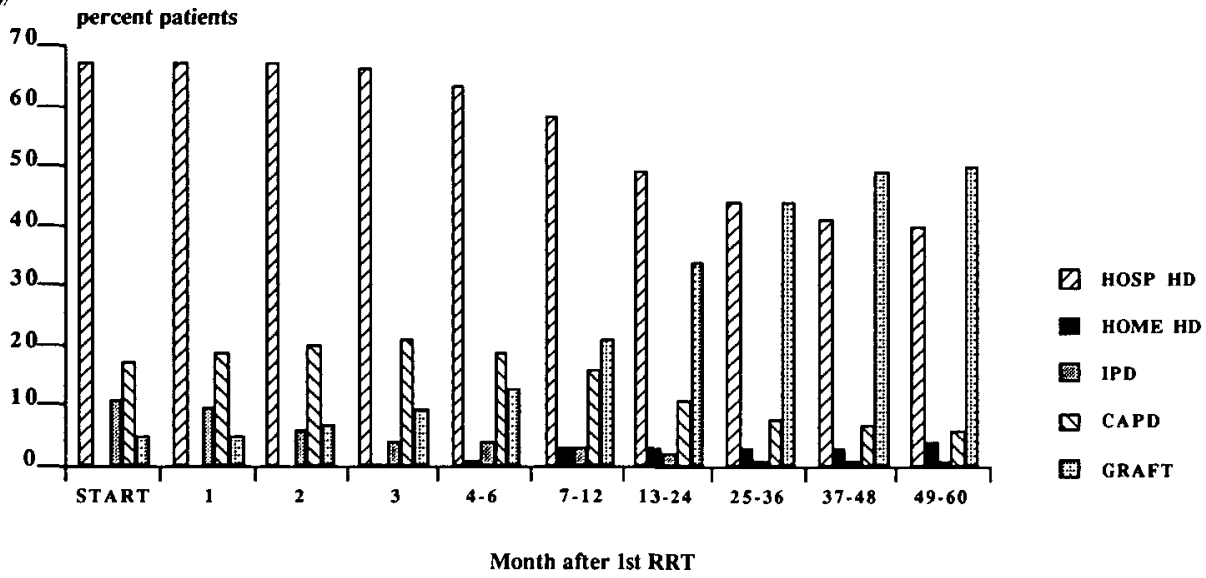

Fig. 4. Proportional distribution of children between modes of RRT at specified intervals over a 60 -month period after start of treatment. Children commencing treatment in the years 1980-1986 included in the analysis.

$45 \%$ of treatment, home haemodialysis $3 \%$, and CAPD $8.5 \%$.

\section{Characteristics of Children on CAPD in Europe}

The age and sex distribution of paediatric patients who commenced CAPD as first method of treatment in the years 1980-1986 has been analysed. There appears to be no relationship between sex and choice of CAPD as first RRT. The ratio of females to males accepted onto CAPD in the 7 years was 1:1.3 compared to $1: 1.2$ for patients taken onto all forms of RRT over the same period.

The age of 561 children accepted onto CAPD between 1980 and 1986 is given in Fig. 5. Over $40 \%$ of these were aged $10-15$ years and these results are similar

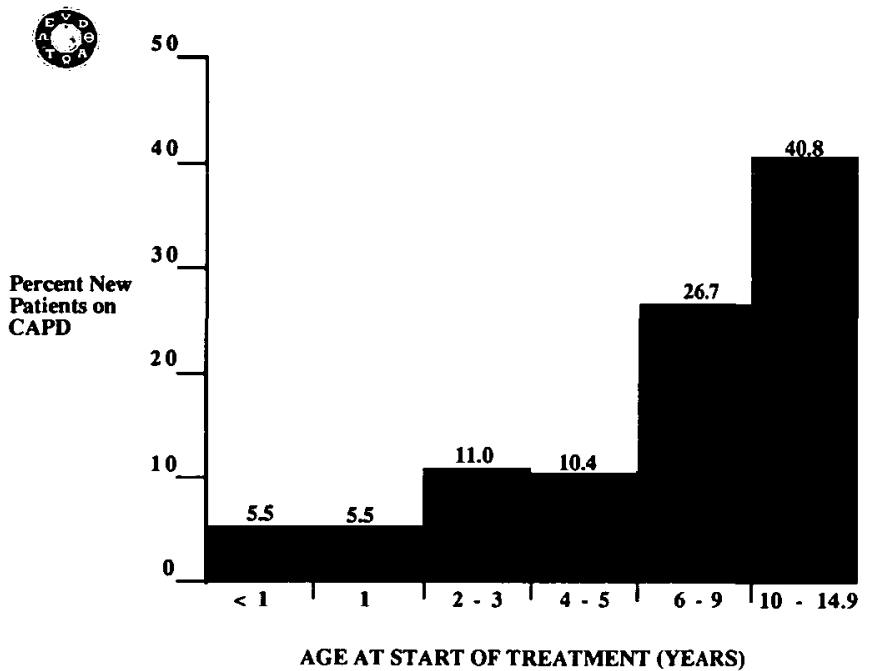

Fig. 5. Age distribution of patients who commenced CAPD as first treatment in the years 1980-1986. to those reported in the same age group in the United States [2]. This reflects the preponderance of older children amongst all those accepted for RRT. Even more interesting is the proportion of children taken onto RRT in each age group who were put onto CAPD as first treatment (Fig. 6). The use of CAPD as first therapy was most popular in those aged less than 6 years at start of treatment, with approximately one-third of these children on CAPD compared to no more than $13 \%$ of those aged $10-14$ years. This pattern of use did not differ between males and females.

Primary renal diseases amongst children treated by CAPD have been compared to those of children commencing on other forms of RRT. There appears to be no relationship between selection of CAPD and the primary renal disease leading to end-stage renal failure.

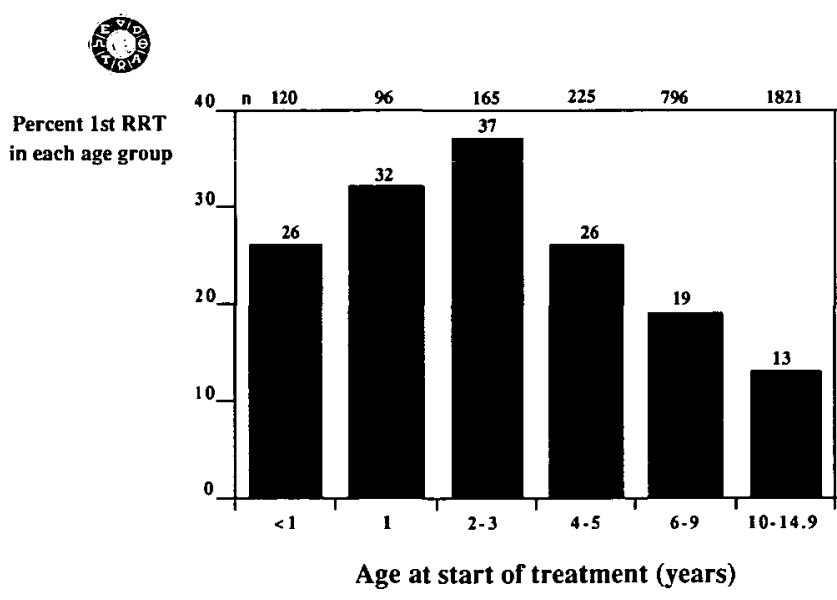

Fig. 6. Proportion of children who were started on CAPD as first therapy according to age at start of treatment between 1980 and 1986. Absolute numbers of paediatric patients commencing treatment in each age group from one year old are also shown (n). 


\section{Causes of Abandonment}

In the past the EDTA Registry has collected information on cause of abandonment of CAPD. This has been studied for children only over the years 1981-1984, and the distribution of causes is shown in Fig. 7. The analysis includes all paediatric patients who abandoned CAPD over the 4-year period, irrespective of whether it was the first or subsequent method of treatment. The causes of abandonment listed by the Registry are: peritonitis, other abdominal complications, inadequate dialysis (including inadequate ultrafiltration), inability to cope with CAPD, patient's request, family's request, and other complications related to CAPD. Information on abandonment between 1981 and 1984 was provided in 817 cases. Half of all abandonments were due to peritonitis, with other abdominal complications accounting for a further $24 \%$. Patients' and families' requests accounted for only a few cases of abandonment over the 4 years.

The Registry analysed the incidence of peritonitis in children on CAPD, and in 19830.15 episodes per patient-month were recorded in paediatric patients. This incidence was not different from that recorded in 1981 and 1982.

The contribution of CAPD to the support of children prior to grafting has been analysed amongst those who commenced renal replacement therapy in 1984 and 1985 and whose first form of treatment was CAPD. There were 193 children for whom the outcome was known 1 year after commencing therapy on CAPD. Of these, 34

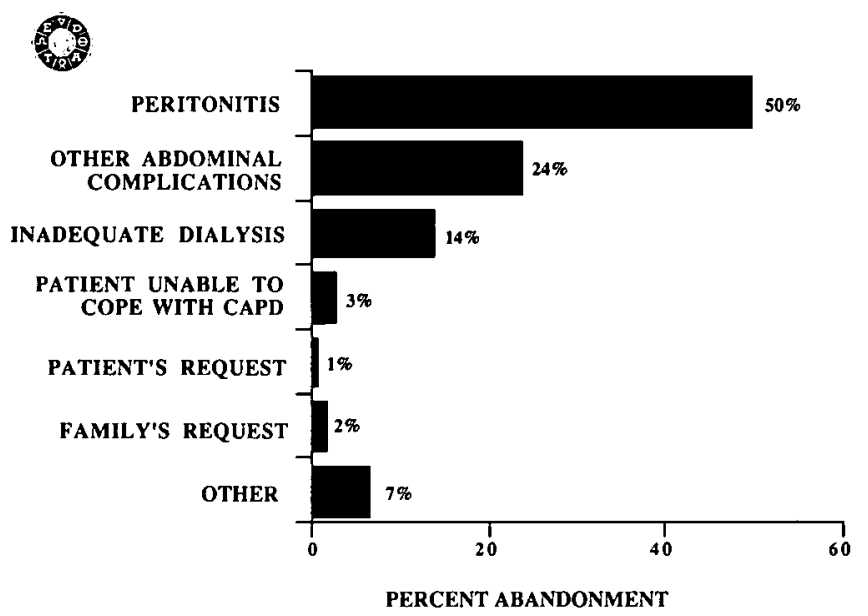

Fig. 7. Proportional distribution of causes of abandonment of CAPD amongst patients who left the treatment between 1981 and 1984 Codes for cause of abandonment:

1. Peritonitis

2. Other abdominal complications

3. Inadequate dialysis

4. Patient unable to cope with CAPD

5. Patient's request

6. Family's request

7. Other complications related to CAPD.
(17.6\%) had either moved to another form of dialysis or died and a further $51(26.4 \%)$ had received a transplant. Six cases $(3.1 \%)$ had recovered function of their own kidneys. If transplantation is not considered as a failure of support and is computed as a loss to follow-up (i.e. such cases are not at risk of failure of support on CAPD), a CAPD support rate of $79.2 \%$ after the first year is calculated using actuarial methods.

\section{Causes of Death Among Children on CAPD}

From 1980 to 1986 there were 54 deaths recorded in paediatric patients on CAPD, compared to a total of 386 for all other forms of RRT. The proportion of deaths due to seven different groups of causes is shown according to last method of treatment in Fig. 8. Care must be taken in making any comparison between treatments because it is difficult to assess the contribution of a mode of therapy to the cause of death. The patients have been divided according to last treatment but this does not necessarily reflect the total contribution of that treatment to the maintenance of the patient while alive. Amongst children whose last method of treatment was CAPD, the highest proportion of deaths were reported as due to cardiac causes $(30 \%)$ and infectious causes $(20 \%)$. In patients on other forms of RRT, cardiac causes accounted for $46 \%$ of mortality and infections only $17 \%$.

In conclusion, the data collected by the EDTA-ERA Registry show that continuous peritoneal dialysis is increasingly used in Europe as a method of treatment of ESRF in children. Important differences exist among various European countries in the use of this mode of RRT; the reasons for this are multiple and most prob-

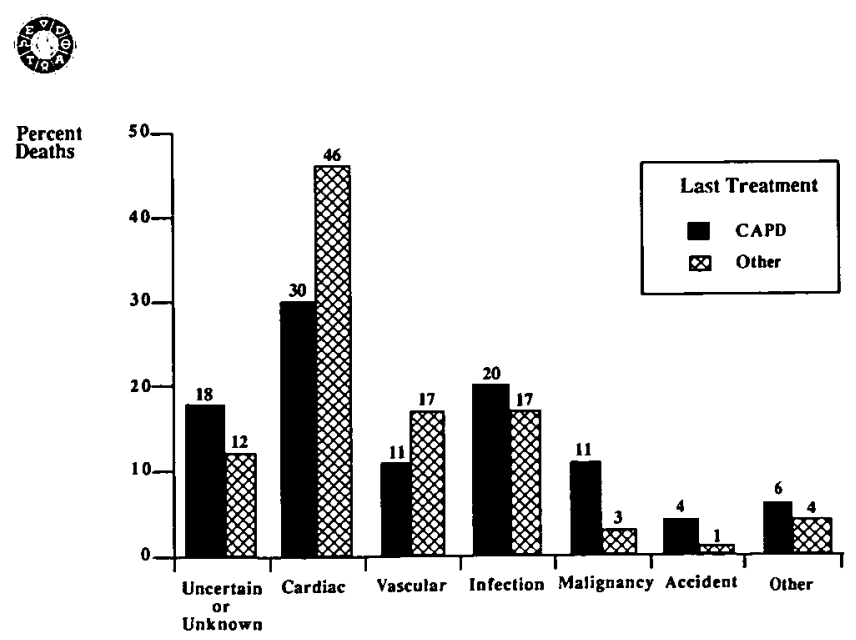

Fig. 8. Proportional distribution of causes of death amongst children who died between 1980 and 1986 according to last method of treatment. 
ably vary not only from one country to another, but also from one centre to another.

Acknowledgement. The work of all the doctors and their staff who completed the EDTA questionnaires is gratefully acknowledged.

\section{References}

1. Arbus GS. Paediatric patients in 1985. In Canadian Renal Failure Register, 1985 Report. Kidney Foundation of Canada, 1986: 87-109

2. Novello AC, Lindblad AS, Novak JW, Nolph KD. Demographic data on the use of CAPD/CCPD as a primary dialytic therapy in children in the United States. In Fine RN, ed. Chronic Ambulatory
Peritoneal Dialysis (CAPD) and Chronic Cycling Peritoneal Dialysis (CCPD) in Children, Martinus Nijhoff, Boston, 1987: 13-20

3. Ehrich JHH, Rizzoni G, Broyer $M$ et al. Combined report on regular dialysis and transplantation of children in Europe, 1987. Nephrol Dial Transplant 1989; 4 [Suppl 2]: 33-41

4. Broyer M, Brunner FP, Brynger $H$ et al. Demography of dialysis and transplantation in Europe, 1984. Nephrol Dial Transplant 1986; $1: 1-8$

5. Golper TA, Geerlings W, Selwood NH et al. Peritoneal Dialysis results in the EDTA Registry. In Nolph KD, ed. Peritoneal Dialysis. Kluwer, Netherlands, 1989: 414-428

6. Kramer P, Broyer M, Brunner FP et al. Combined report on regular dialysis and transplantation in Europe, XIV, 1983. Proc EDTA-ERA 1985; 21: 2-65

7. Broyer M, Rizzoni G, Brunner FP et al. Combined report on regular dialysis and transplantation of children in Europe, XIV, 1984. Proc EDTA-ERA 1985; 22: 55-79

Received for publication 1.5 .90

Accepted in revised form 10.8 .90 